\section{Risk of unsuccessful noninvasive ventilation for acute respiratory failure in heterogeneous neuromuscular diseases: a retrospective study} Kaoru Kinugawa, Yuto Uchihara, Hiroya Ohara, Nobuyuki Eura, Ryogo Syobatake, Nobuhiro Sawa, Kiriyama Takao, Kazuma Sugie, Satoshi Ueno

Department of Neurology, Nara Medical University, Kashihara, Japan

\begin{abstract}
If invasive ventilation can be avoided by performing noninvasive mechanical ventilation (NIV) in patients with acute respiratory failure (ARF), the disease can be effectively managed. It is important to clarify the characteristics of patients with neuromuscular diseases in whom initial NIV is likely to be unsuccessful. We studied 27 patients in stable neuromuscular condition who initially received NIV to manage fatal ARF to identify differences in factors immediately before the onset of ARF among patients who receive continuous NIV support, patients who are switched from NIV to invasive ventilation, and patients in whom NIV is discontinued. Endpoints were evaluated 24 and 72 hours after the initiation of NIV. After 24 hours, all but 1 patient with amyotrophic lateral sclerosis (ALS) received continuous NIV support. 72 hours later, 5 patients were switched from NIV to invasive ventilation, and 5 patients continued to receive NIV support. 72 hours after the initiation of NIV, the proportion of patients with a diagnosis of ALS differed significantly among the three groups $(\mathrm{P}=0.039)$. NIV may be attempted to manage acute fatal respiratory failure associated with neuromuscular diseases, but clinicians should carefully manage the clinical course in patients with ALS.
\end{abstract}

\section{Introduction}

Noninvasive mechanical ventilation (NIV), including noninvasive positive pressure ventilation (NIPPV), is a well-accepted treatment for chronic neuromuscular respiratory failure associated with conditions such as amyotrophic lateral sclerosis, and NIV prolongs survival. ${ }^{1}$ However, acute respiratory failure (ARF) frequently requires invasive ventilation. Clinicians are often pressed for an immediate decision as to whether NIV or invasive ventilation should be used in patients with various neuromuscular diseases who suddenly present with potentially fatal ARF. If invasive ventilation can be avoided by performing NIV in patients with ARF, the severity of disease can be effectively managed, and the outcomes of neuromuscular diseases can be improved because invasive ventilation requires endotracheal intubation or tracheotomy, which can cause serious complications, especially in patients with respiratory infection. In contrast, NIV carries a lower risk of respiratory infection. ${ }^{2}$ However, the usefulness of NIV for ARF has been limited. It is important to clarify the characteristics of patients with neuromuscular diseases in whom initial NIV is likely to be unsuccessful, leading to the need for invasive ventilation. We studied 27 patients in stable neuromuscular condition who initially received NIV to manage fatal ARF to identify differences in factors immediately before the onset of ARF among patients who receive continuous NIV support, patients who are switched from NIV to invasive ventilation, and patients in whom NIV is discontinued.

\section{Materials and Methods}

We retrospectively studied 27 medically stable patients with neuromuscular diseases who presented with ARF that required mechanical ventilation to save their lives and to relieve respiratory distress despite oxygen administration. Patients with other causes of pre-ventilatory respiratory failure, such as chronic obstructive pulmonary disease or lung cancer, were excluded. None of the patients had a history of NIV, endotracheal intubation, tracheotomy, or invasive ventilation, and all patients initially received NIV after the onset of ARF. All patients other than those with coma had severe respiratory distress. A decrease in consciousness level was evident in 16 patients, and coma status as defined as a score of $\geq 100$ on the Japan Coma Scale ${ }^{3}$ was seen in 6 patients. Twenty-one patients were urgently admitted to our hospital, and the condition of 4 other medically stable patients suddenly worsened during hospitalization. Two other patients were admitted to our hospital within 23 hours from presentation. Before the initiation of NIV, 26 patients were given the following diagnoses: probable multiple systemic atrophy (MSA) according to the Gilman criteria ${ }^{4}$ in 5 patients, clinically definitive Parkinson's disease (PD) according to the UK
Correspondence: Hiroshi Kataoka, Department of Neurology, Nara Medical University, 840 Shijo-cho, Kashihara, Nara 634-8522, Japan.

Tel: +81.744.29.8860 - Fax: +81.744.24.6065 E-mail: hk55@naramed-u.ac.jp

Key words: Noninvasive mechanical ventilation; Acute respiratory failure; Amyotrophic lateral sclerosis; Risk.

Contributions: HK was responsible for the overall study design, wrote the manuscript, contributed to organization, planning and coordination of the study, and contributed to analysis and interpretation of data; $\mathrm{HK}, \mathrm{HN}$, KK, UU, HO, NE, RS, NS, TK, and KS contributed to running the study and acquisition of data; HK and SU contributed to drafting and critical revision of part of the submitted materials.

Conflict of interest: the authors declare no potential conflict of interest.

Received for publication: 23 September 2016. Revision received: 5 February 2017.

Accepted for publication: 6 February 2017.

This work is licensed under a Creative Commons Attribution NonCommercial 4.0 License (CC BY-NC 4.0).

C Copyright H. Kataoka et al., 2017

Licensee PAGEPress, Italy

Neurology International 2017; 9:6904

doi:10.4081/ni.2017.6904

Parkinson's Disease Society Brain Bank criteria $^{5}$ in 2 patients, clinically probable or definite amyotrophic lateral sclerosis (ALS) fulfilling the El Escorial revised criteria ${ }^{6}$ in 8 patients, myotonic dystrophy (MyD) in 6 patients, polymyositis (PM) in 1 patient, both myositis and myasthenia gravis in 1 patient, $\mathrm{MG}$ in 2 patients, and chronic inflammatory demyelinating polyneuropathy in 1 patient. Human herpes virus-6 encephalitis was diagnosed on the basis of polymerase chain reaction after starting NIV in 1 patient.

\section{Factors evaluated immediately before the initiation of noninvasive mechanical ventilation}

Arterial blood gas (ABG) analysis was performed within a short period before starting NIV (median 68 minutes). At the same time, the presence of coexistent pulmonary disease such as aspiration pneumonia, the Japan Coma Scale score, ${ }^{3}$ and the need for oxygen administration were evaluated. Pneumonia was diagnosed on chest radiography and computed topography. The modified ranking scale score or bulbar 
symptoms were evaluated when the patient was in medically stable condition within 1 month before the initiation of NIV.

\section{Endpoints and statistical analysis}

Endpoints were evaluated 24 hours and 72 hours after the initiation of NIV. Each endpoint was judged as three states (persistent NIV support, transition from NIV to invasive ventilation, and free of mechanical ventilation). Variables that were not normally distributed were transformed to natural logarithms or categorical quartile groups. Normally distributed variables are presented as means $\pm \mathrm{SD}$, and asymmetrically distributed variables are presented as medians with interquartile ranges (IQR). The statistical significance of differences in pre-ventilation clinical factors among these three groups was analyzed by linear regression analysis. SPSS software (Version 18) was used to perform statistical analysis. We obtained their informed consents of all patients as well as from their families about the clinical procedure including NIV. As for the patients with compromised cognitive functions, we obtained the informed consent from their families. The protocol of this study was approved by the medical ethics committee of Nara Medical University.

\section{Results}

Detailed clinical information, including the results of $\mathrm{ABG}$ analysis in each patient, is shown in Supplementary Table 1. After 24 hours, all but 1 patient with ALS received continuous NIV support. Seventytwo hours later, 5 patients were switched from NIV to invasive ventilation, and 5 patients continued to receive NIV support (Figure 1). Seventy-two hours after the initiation of NIV, the proportion of patients with a diagnosis of ALS differed significantly among the three groups $(\mathrm{P}=0.039)$, and other variables, including $\mathrm{ABG}$, did not differ (Table 1). One week later, two patients (1 with PD and 1 with MyD) died, 5 patients received invasive ventilation, and 4 patients were free of ventilation. One month later, 8 patients underwent invasive ventilation or died, and 6 patients were free of ventilation. As for 8 patients with ALS, 3 patients received invasive mechanical ven-

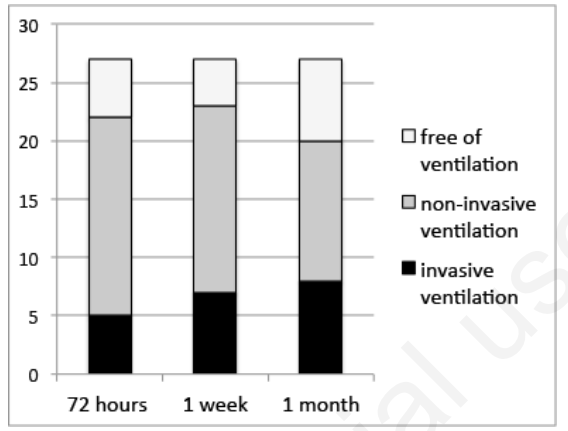

Figure 1. The number of patients with neuromuscular diseases who had acute respiratory failure and received continuous noninvasive mechanical ventilation (NIV) support, number of patients who required invasive ventilation, and that of patients who could discontinue NIV. tilation after 72 hours. Although statistical significance was not reached, hypercapnia before starting NIV was frequent among ALS patients with unsuccessful NIV (Figure 2).

\section{Discussion}

The present study found that medically stable patients with ALS who initially received NIV tended to require invasive ventilation after NIV. One retrospective study that evaluated 76 patients who had various neuromuscular diseases associated with ARF, including 10 patients with ALS

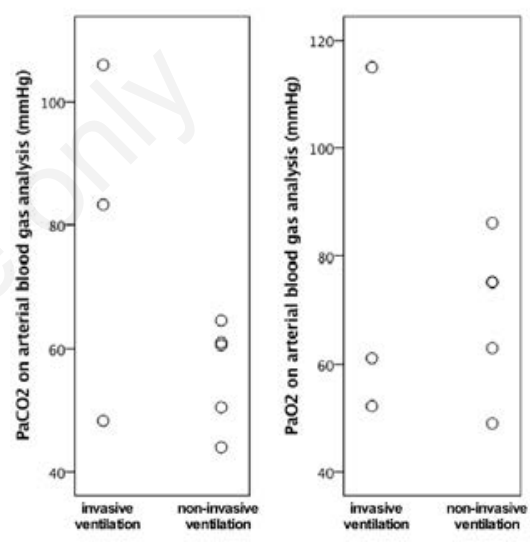

Figure 2. Carbon dioxide (PaCO2) and oxygen $(\mathrm{PaO} 2)$ on arterial blood gas analysis before starting noninvasive mechanical ventilation.

Table 1. Linear regression analysis of differences in clinical factors before the onset of acute respiratory failure among patients who received continuous noninvasive mechanical ventilation support, those who were switched from noninvasive to invasive ventilation, and those in whom noninvasive mechanical ventilation was discontinued.

\begin{tabular}{|c|c|c|c|c|}
\hline & Free of ventilation $(n=5)$ & Non-invasive ( $\mathrm{n}=17$ ) & Invasive $(\mathrm{n}=5)$ & $\mathbf{P}$ \\
\hline Age (mean) & $64.2,13.1$ & $64,15.0$ & $63.2,14.8$ & 0.913 \\
\hline Gender, male & 3,60 & $11,64.7$ & 4,80 & 0.521 \\
\hline Amyotrophic lateral sclerosis (n) & 0,0 & $5,29.4$ & 3,60 & $0.039 *$ \\
\hline History of cardiopulmonary disease (n) & 2,40 & $4,23.5$ & 2,40 & 1 \\
\hline Bulbar symptoms (n) & 3,60 & $9,52.9$ & 2,40 & 0.545 \\
\hline Modified ranking scale (mean, SD) & $3.8,1.3$ & $3.5,1.1$ & $3.4,0.8$ & 0.604 \\
\hline \multicolumn{5}{|l|}{ At initiation of non-invasive ventilation } \\
\hline Coma state (JCS 300) (n) & 1,20 & $5,29.4$ & 0,0 & 0.466 \\
\hline Coexistence with pulmonary disease (n) & 4,80 & $8,47.0$ & 1,20 & 0.061 \\
\hline Oxygen (n) & 5,100 & $8,47.0$ & 4,80 & 0.531 \\
\hline $\mathrm{Ph}$ (mean, SD) & $7.37,0.06$ & $7.33,0.11$ & $7.35,0.09$ & 0.715 \\
\hline $\mathrm{PaO2}$ (median) & $97.6(60.9,99.7)$ & $67.5(53.9,75.2)$ & $63.1(61.1,79.1)$ & 0.712 \\
\hline Pac02 (average) & $63.42,63.8$ & $66.48,61.7$ & $72.82,71.6$ & \\
\hline Log-transformed (mean, SD) & $4.08,0.44$ & $4.15,0.34$ & $4.26,0.31$ & 0.426 \\
\hline HNO3 (mean, SD) & $34.72,10.99$ & $33.5,6.28$ & $31.46,4.53$ & 0.464 \\
\hline Sp02 (median) & $97.1(94.0,98.8)$ & $90.1(87.0,94.9)$ & $95.3(91.2,97.5)$ & 1 \\
\hline
\end{tabular}

SD, standard deviation; JCS, Japan Coma Scale; PaO2, carbon oxygen; Paco2, carbon dioxide; HNO3, nitric acid; SpO2, pulse oxyhemoglobin saturation. ${ }^{*} \mathrm{P}<0.05$. Data are reported as means (SD), medians (IQR: interquartile range), numbers (\%), or averages (median). 
(13.1\%), reported that 12 patients $(15.7 \%)$ received continuous NIV and 47 patients $(61.8 \%)$ required invasive ventilation or died. 7 In the present study, including 8 patients with ALS (29.6\%), 12 patients (44.4\%) received continuous NIV support and 7 patients $(25.9 \%)$ required invasive ventilation or died. These results are inconsistent with the findings of previous studies, possibly because of different proportions of patients with ALS, much longer endpoints than those in the present study, or different definitions of endpoints. ${ }^{7}$ However, the proportion of patients who required invasive ventilation or died increased after 1 month (Figure), similar to the results of a previous study. ${ }^{7}$

We found no prognostically significant pre-ventilation values on ABG analysis. In a previous study, lower $\mathrm{pH}$ and $\mathrm{PO} 2$ values and higher $\mathrm{PCO} 2$ values before ventilation were associated with poor functional outcomes at discharge, and bicarbonate $>30 \mathrm{mg} / \mathrm{dL}, \quad \mathrm{PCO} 2>50 \mathrm{mmHg}$, and $\mathrm{pH}<7.30$ were associated with an increased risk of death in survivors with neuromuscular diseases. ${ }^{7}$ These trends were seen in the present study. Seventy-two hours after start- ing NIV, all patients with unsuccessful NIV had a value of PCO2 $>50 \mathrm{mmHg}$, and bicarbonate $>30 \mathrm{mg} / \mathrm{dL}$ and $\mathrm{pH}<7.30$ were seen in 4 patients and 3 patients with unsuccessful NIV, respectively.

\section{Conclusions}

In the present study, some patients were free of mechanical ventilation. However, mechanical ventilation was needed in a heterogeneous cohort of 22 patients with neuromuscular diseases.

\section{References}

1. Pinto AC, Evangelista T, Carvalho M, et al. Respiratory assistance with a noninvasive ventilator (Bipap) in MND/ALS patients: survival rates in a controlled trial. J Neurol Sci 1995;12: 19-26.

2. Hillberg RE, Jhonson DC. Noninvasive ventilation. New Engl J Med 1997;337: 1746-52.
3. Takagi K, Aoki M, Ishii T, et al. Japan Coma Scale as a grading scale of subarachnoid hemorrhage: a way to determine the scale. No Shinkei Geka 1998;26:509-15.

4. Gilman S, Wenning GK, Low PA, et al. Second consensus statement on the diagnosis of multiple system atrophy. Neurology 2008;71:670-6.

5. Hughes AJ, Daniel SE, Kilford L, Lees AJ. Accuracy of clinical diagnosis of idiopathic Parkinson's disease: a clinico-pathological study of 100 cases. J Neurol Neurosurg Psychiatry 1992;55: 181-4.

6. Brooks BR, Miller RG, Swash M, Munsat TL. World Federation of Neurology Research Group on Motor Neuron Diseases: El Escorial revisited: revised criteria for the diagnosis of amyotrophic lateral sclerosis. Amyotroph Lateral Scler Other Motor Neuron Disord 2000;1:293-9.

7. Cabrera Serrano M, Rabinstein AA. Usefulness of pulmonary function tests and blood gases in acute neuromuscular respiratory failure. Eur $\mathrm{J}$ Neurol 2012;19:452-6. 